\begin{tabular}{l|l} 
REVISTA & $\begin{array}{l}\text { Revista Educación } \\
\text { ISSN: 0379-7082 } \\
\text { ISSN: 2215-2644 } \\
\text { revedu@gmail.com } \\
\text { Universidad de Costa Rica } \\
\text { Costa Rica }\end{array}$
\end{tabular}

\title{
La necesidad de educar en perspectiva de género
}

\author{
Jiménez Quenguan, Myriam; Galeano Barbosa, Deison Julián \\ La necesidad de educar en perspectiva de género \\ Revista Educación, vol. 44, núm. 1, 2020 \\ Universidad de Costa Rica, Costa Rica \\ Disponible en: http://www.redalyc.org/articulo.oa?id=44060092035 \\ DOI: https://doi.org/10.15517/revedu.v44i1.38529
}

Esta obra está bajo una Licencia Creative Commons Atribución-NoComercial-SinDerivar 3.0 Internacional. 


\title{
La necesidad de educar en perspectiva de género
}

The Need for Gender Perspective when Educating

Myriam Jiménez Quenguan

Universidad Santo Tomás, Colombia

myriamjqsigloxxi@gmail.com

(iD http://orcid.org/0000-0003-0807-6037.

Deison Julián Galeano Barbosa

Universidad Santo Tomás, Colombia

deisongaleano12@gmail.com

iD http://orcid.org/0000-0002-4618-885X

\author{
DOI: https://doi.org/10.15517/revedu.v44i1 1.38529
http://www.redalyc.org/articulo.oa?id $=44060092035$ \\ Redalyc: http://www.redalyc.org/articulo.oa?id=44060092035
}

Recepción: 12 Agosto 2019

Aprobación: 04 Diciembre 2019

\section{RESUMEN:}

Este artículo de revisión bibliográfica, indaga en la problemática de género que desarrolló el proyecto de la Universidad Santo Tomas titulado: Yo soy tú, tú eres todos. Somos letras vivas en movimiento: un acercamiento lúdico infantil en perspectiva de género (Jiménez, 2018). Acorde con la misión educativa y humanizadora, el estudio se organizó en comunidad y ejerció desde la educación una influencia positiva en el contexto. Aquí se toma como eje central el tema de género, a fin de reflexionar críticamente para construir pedagogías que propicien el autoconocimiento de la subjetividad y el debate frente a algunos de sus flagelos, a fin de valorar y respetar la vida desde la niñez. La metodología incluye exploración de fuentes recientes; la búsqueda y sistematización se orientó acorde a los propósitos del proyecto: caracterizar la población infantil y su subjetividad de género; identificar problemáticas de género, a través de historias y acontecimientos importantes de vida; y, proponer una estrategia didáctica lúdica cercana. Los resultados permiten concluir que es necesario seguir trabajando en perspectiva de género desde la infancia y, profundizar en violencias de género recurrentes para lograr, a través de la educación, un impacto favorable desde la inclusión, la justicia y la equidad de género.

Palabras Clave: Género, Infancia, Subjetividad, Educación, Violencia infantil.

\section{ABstract:}

This bibliographic review explores gender-related issues in a study conducted by the Universidad Santo Tomás (St. Thomas University) entitled: Yo soy tú, tú eres todos (I am you; You are everyone). We are living and moving languages: approaching children at play with a gender perspective (Jiménez, 2018). The study, which was organized within a community. A gender focus was used to critically reflect on the development of pedagogies to foster self-knowledge of subjectivity and discussion in order to value and respect life from childhood. The methodology includes exploration of recent sources; data searches and systematization were commensurate with the project endpoint: characterization of the child population and its gender subjectivity; identifying gender issues through stories and important life events, and, propose a childlike, didactic strategy. Based on the results, we need to continue to work on gender perspectives and deepen recurrent gender violence to favorably impact inclusion, justice and gender equality.

KEYwORDS: Gender, Childhood, Subjectivity, Education, Child Violence.

Se equivocó el Muñeco: la niña era intocable y era sagrada.

No podia profanarla y salir impune.

Lo sagrado calcina a quien lo toca.

Fuente: Laura Restrepo, Los Divinos 


\section{INTRODUCCIÓN A LA PROBLEMÁTICA}

En países como Colombia los índices de maltrato y violencia infantil son alarmantes, así lo reportan los recientes datos del Instituto Nacional de Medicina Legal y Ciencias Forenses [INMLCF], en la publicación Masatugó (Heredia, 2015), donde las cifras epidemiológicas de violencia contra niñas y adolescentes mujeres en el periodo 2009-2014, ascendieron a 39.502 casos. El grupo etario más vulnerable fue el comprendido entre 10 a 14 años, el grado escolar más afectado fue el de básica primaria, la población más afectada fue la campesina y los principales agresores los padres de familia (Heredia, 2015) Este problema se presenta en distintos países, pero se agudiza en aquellos en vías de desarrollo, allí el fenómeno de la violencia y sus distintas tipificaciones se incrementa en los sectores más vulnerables: las mujeres, las niñas y los niños, las causas son multifactoriales.

No es posible la paz si no se visibilizan y reparan a sus víctimas, en Colombia los niños han padecido el estigma del abuso hasta límites insospechados. Entre algunos recientes casos de feminicidios infantiles están los de Juliana Andrea Samboni Muñoz (Caracol, 2016), niña de origen indígena y humilde, menor de 7 años, secuestrada, torturada, violada y asesinada el 4 de diciembre de 2016 por el acaudalado arquitecto Rafael Uribe Noguera en una zona de Chapinero alto en Bogotá; Sara Ayolima Salazar Palacios (Espectador, 2017), niña de 3 años abusada física y sexualmente por su padrino Edilberto Rojas Torres en complicidad con su madrina, Ángela Yohana Guerra Ureña y las autoridades locales, en la vereda El Palmar, de Armero Guayabal (norte del Tolima), antes de morir la niña estuvo varias veces hospitalizada por anemia y desnutrición, por picadura de animal y ojos morados, el 22 de abril de 2017 llegó con heridas graves que le ocasionaron la muerte, el parte médico comprueba signos de tortura física y sexual; Erika Tatiana González (Espectador, 2018), niña de seis años, oriunda del Meta, su cuerpo sin vida fue hallado en bolsas de plástico negro, el 24 de noviembre de 2018 en el río Guaviare en Mapiripán, Meta, el responsable de su muerte y abuso sexual fue un menor de 16 años que conocía a la víctima. Según lo informa la redacción del periódico Espectador (2018), con base en las cifras reportadas por el Instituto Nacional de Medina Legal y Ciencias Forenses, hasta septiembre de 2018, se habían cometido en Colombia 722 feminicidios.

A la anterior y aterradora realidad se suman las constantes violencias de género ejercidas contra las niñas. Según estudios recientes, existen representaciones de género que promueven diversas violencias contra la mujer, nociones de poder masculino que acrecientan el fenómeno del feminicidio. "El feminicidio es un fenómeno que afecta a todos los países a nivel mundial y que, además, está en aumento; en los países de América Latina, en particular, las cifras hablan por sí mismas" (Cardona y Onofre, 2017, pp. 531-532) Y aunque Colombia a través de la Ley 1761 de 2015 (Presidencia, 2015) lo tipifica como delito, hace falta visibilizar mejor los casos de feminicidios infantiles, este problema se recrudece si no existe una educación en género y un sistema de justicia eficaz porque la mayoría de los delitos tienden a quedar impunes. ONU Mujeres (2018) señala que los feminicidios se han convertido en una pandemia mundial y según lo reporta el INMLCF (2017) en Forensis Datos para la vida, las mujeres siguen siendo las víctimas más agredidas y en lo que respecta a los menores:

\footnotetext{
La violencia contra niños, niñas y adolescentes (NNA), durante el año 2018 mantuvo la tendencia a tener mayoría de víctimas de sexo femenino, en edad adolescente, y con bajo nivel de escolaridad. La tendencia de que los padres y madres son los mayores agresores se ha mantenido. La mayor parte de los casos de violencia contra NNA se dieron en el hogar y en las cabeceras municipales. Las ciudades donde ocurrieron la mayoría de los casos siguen un orden similar al de años anteriores (Rodríguez y Jiménez, 2018, p. 139).
}

Hasta 2018 en Colombia las cifras iban en aumento, cada día eran asesinadas aproximadamente por lo menos tres mujeres, de “ 404 casos de asesinatos de mujeres registrados en el año 2018, 78 fueron catalogados bajo la circunstancia feminicidio” (Lozano, 2018, p. 72) Pero, en este año señala Lozano, gracias a la implementación de los Acuerdos de Paz con las FARC las tasas se redujeron. Sin embargo, es necesario distinguir en el caso de las mujeres, el homicidio del feminicidio que en el libro Femicide. The politics of 
Woman Killing, editado por Radford y Rusell (1992), lo definieron como la muerte de mujeres por parte de hombres por el hecho de ser mujeres. Esta verdad exige una gran responsabilidad, por esto, a nivel educativo es un imperativo, trabajar en perspectiva de género. El objetivo de esta revisión es propiciar un debate de género desde un acercamiento crítico; la búsqueda se efectuó a partir de los años noventa hasta la actualidad, teniendo en cuenta varias bases de datos como Scopus, Publindex, Dialnet y Google Shcolar. Se integraron tres aspectos: la relación entre el contexto y la subjetividad de género, las problemáticas de género en las poblaciones infantiles y el género en las narraciones e historias de vida.

\section{ConTEXTO Y SUBJETIVIDAD DE GÉNERO:}

\subsection{La irreductibilidad de la palabra género}

Para entender el concepto de género, es de destacar la publicación del artículo de Scott (1990), titulado El género: una categoria útil para el análisis histórico, la autora presenta cómo inicialmente existía un interés puramente histórico, pero desde allí se ven vestigios de lo que será la posterior y más reciente manera de tratarlo; su aporte permite analizar la problemática de género desde el sentido histórico-contextual del concepto en cuestión. Inicialmente Scott cuestiona a la tradicional concepción de género, la cual se limita a entenderlo como la manifestación de lo masculino y lo femenino, negando la posibilidad de integrar otras opciones sexuales humanas. Hasta hace poco tiempo en los diccionarios de casi todas las lenguas como el Fowler's Dictionary of Modern English Usage (Butterfield, 2015) interpretaban género como un término estrictamente gramatical para hablar de personas o criaturas del género masculino y femenino. Scott (1990) recuerda que en su acepción más reciente aparece la de las feministas estadounidenses, quienes rechazaron una lectura estrictamente biologicista que lo relacionaba con sexo o diferencia sexual, destacando que género también expresa varias formas de la feminidad.

Es decir, la palabra género es problemática y su significado aún está en construcción. Para Scott (1990), esto implica no solo abordar la historia de las mujeres sino también la de los hombres y demás grupos de género, al citar a Natalie Zemon Davis (1975) afirma: "Nuestro propósito es descubrir el alcance de los roles sexuales y del simbolismo sexual en las diferentes sociedades y periodos, para encontrar qué significado tuvieron y cómo funcionaron para mantener el orden social o para promover su cambio" (p. 4). Por tanto, hablar de género implica revisar diversos estudios y posturas feministas, ampliar la visión historicista y reescribir una historia plural e incluyente que dé cuenta, por un lado, de las experiencias de mujeres, población que ha estado oprimida desde hace varios milenios y, por otro lado, de la comprensión de sus desigualdades, de su situación socioeconómica y también étnica. Sobre la marginalidad de las mujeres existen muchos estudios y dada su prolongada exclusión histórica, en los últimos años casi todos los gobiernos del mundo incluyen en sus políticas el tema de género; se ha pasado de un mínimo interés a uno de carácter amplio. Sin embargo, sigue siendo prioritario incluirlo en todos los ámbitos, principalmente en la educación, porque aún en pleno siglo XXI existen problemas de género como el feminicidio y la Violencia Basada en Género VBG que posee un sinnúmero de tipificaciones (violencia física, psicológica, verbal, laboral, jurídica, etc.).

Hablar de género sigue siendo complejo, como fenómeno social implica ir más allá de la mirada historicista o política, exige comprender las realidades de género, sus búsquedas, su subjetividad, sus relaciones, sus expresiones.

Su uso explícito rechaza las explicaciones biológicas, del estilo de las que encuentran un denominador común para diversas formas de subordinación femenina en los hechos de que las mujeres tienen capacidad para parir y que los hombres tienen mayor fuerza muscular. En lugar de ello, género pasa a ser una forma de denotar las 'construcciones culturales', la creación totalmente social de ideas sobre los roles apropiados para mujeres y hombres (Scott, 1990, pp. 6-7). 
Scott señala que la definición de género fue impuesta sobre un cuerpo sexuado, palabra limitada a la práctica, a los roles y a la diferencia sexual, pero, su uso es mucho más amplio, pues se refiere a estructuras e ideologías que afectan a los niños, las familias, los contextos y la sociedad en general. Como consecuencias se han desarrollado múltiples lecturas y teorías relacionadas con el género, a nivel femenino se habla, por ejemplo, del feminismo de la igualdad y del feminismo de la diferencia.

Frente a lo anterior, Scott (1990) plantea la necesidad de cambiar el sistema dual: hombre-mujer. La crítica al binarismo sexual, desde finales del siglo XX hasta la actualidad ha ido tomando fuerza. En su libro Microfísica del poder, Foucault (1994), expresa que la sexualidad y por ende el género, son el resultado de un contexto histórico y las relaciones de poder desiguales, donde existe un dominio tradicionalmente patriarcal. La autora recuerda que existen teorías feministas marxistas, psicoanalíticas, lacanianas, donde el lenguaje es fundamental para entender la identidad de género y su subjetividad. Lo cierto es que esta categoría es inestable, no es fija, es cambiante, obedece a un proceso que encierra muchos matices y diferencias. La idea de masculino y de lo femenino se relativiza, no se trata solo de opuestos, importa el contexto. El género está en constante construcción, al no reducirse a la lectura binaria, exige una deconstrucción, una desjerarquización, una crítica de cómo se ha venido entendiendo, además de su confusión epistemológica. Después de su interpretación biologicista e historicista, a finales del siglo XX, surge como categoría analítica, lo que conlleva a hablar de él como un sistema de relaciones sociales y sexuales.

Scott (1990) sostiene:

El núcleo de la definición reposa sobre una conexión integral entre dos proposiciones: el género es un elemento constitutivo de las relaciones sociales basadas en las diferencias que distinguen los sexos y el género es una forma primaria de relaciones significantes de poder (p. 23).

Los cambios sociales corresponden a los de poder. Para Scott (1990), ninguno de los elementos señalados opera sin los demás, así se encuentran:

- Símbolos culturales (por ejemplo, los de la tradición judeocristiana occidental, mitos, etc.)

- Normas y conceptos que interpretan esos símbolos (que se expresan a través de la religión, la educación, las leyes, la política)

- Relaciones de género (parentesco, relaciones familiares, de trabajo, educativas, políticas, económicas, de poder)

- La identidad subjetiva, (influencia biológica y lo cultural).

Estas relaciones contribuyen al análisis concreto de clases, etnicidad o cualquier proceso social. Por consiguiente, para Scott (1990), es necesario pensar el efecto del género en las relaciones humanas y la cultura, pues su función legitimadora opera de muchas maneras. Su uso político también influye en su interpretación, sugiriendo modelos de ser y de actuar.

En la actualidad la visión de género es relativa, está en construcción, el Diccionario de la Real Academia de la Lengua Española, en unas de sus recientes acepciones lo entiende como: "Conjunto de seres que tienen uno o varios caracteres comunes [...] Conjunto de todas las personas." (Real Academia Española [RAE], 2019, letra G) Para la Red Colombiana de Mujeres Científicas (RCMC), nacida en 2015, liderada por la Dra. Ángela Camacho Guzmán, también es importante incluir el análisis de la categoría de género en todas las disciplinas científicas y unir esfuerzos para visibilizar afirmativamente a la mujer. La RCMC (2018) considera que en el mundo contemporáneo es necesaria la inclusión de la categoría de género también al referirse a la actividad científica, investigativa y tecnológica, porque la tradición en el campo del conocimiento, se ha caracterizado por el predominio androcentrista.

El análisis de género debe impactar en todas las esferas humanas, pero sigue siendo problemático. Resulta importante desde un primer momento hacer hincapié en una nueva manera de abordarlo, y así se ha hecho, cuando desde la primera parte del siglo XX y especialmente en los años sesenta, autoras como Simone de 
Beauvoir (1999, 2000), gracias a sus famosos libros El segundo sexo. Los hechos y los mitos y El segundo sexo. Vol. 2. La experiencia de vida, respectivamente, se convirtió en una de las más importantes teóricas del feminismo. Rechazó el esencialismo biologicista y planteó que la construcción de género, obedece más a aspectos culturales. Su legado es retomado por feministas como Judith Butler, en su también reconocido libro El género en disputa. El feminismo y la subversión de la identidad (2007), la autora desde el contexto norteamericano, reflexiona sobre la normatividad sexual dominante y la relatividad de esta categoría que es, además, una teoría, una ideología y una forma de expresión de la subjetividad humana. Butler confirma que género no se reduce a una lectura de rasgos físicos ni el sujeto se limita a un binarismo de género, este se performa de acuerdo con sus relaciones y a sus prácticas. Porque como lo señala Foucault (1987), en Hermenéutica del sujeto, el sujeto se preocupa de sí mismo "el sí mismo se convierte en el objetivo definitivo y único de la preocupación por uno mismo” (p. 68), el gran problema es y seguirá siendo la construcción del sujeto.

\subsection{El problema de la subjetividad}

Con base en lo anterior, acercarse a la subjetividad de género infantil resulta complejo, porque si el mundo adulto apenas está construyendo su concepción, para los menores de edad, en su primer acercamiento hacia la palabra y lo que se comprende, tiende a reproducir las nociones que encuentran en su contexto inmediato. De allí que sea necesario comenzar desde la niñez a cuestionar las ideas que se han tejido en torno al género, a fin de favorecer una mirada más incluyente y menos sexista. Al indagar en la categoría de género en la infancia, se encontraron estudios como los de Witt (1997), quien en Parental influence on children's socialization to gender, analiza los roles sexuales a partir de las relaciones parentales, la construcción de estereotipos y sesgos que se adoptan en los niños. Expone cómo en el contexto norteamericano, la relación parental se entiende bajo la luz de los prejuicios que se tienen sobre los roles de género que se empiezan a construir a partir de la familia y, se afianzan en el colegio, los medios de comunicación, las relaciones, etc. Desde la década de los 90, la cuestión del género se viene gestando en Estados Unidos como una manera de entender las relaciones sociales e históricas. Este texto permite pensar que, en la construcción de la subjetividad de género, la influencia más cercana para los niños es la de su familia, la del contexto inmediato y las tecnologías mediáticas.

Ahora bien, dichos estudios se seguirán trabajando de manera diversa, pero logran un gran salto en el año de 1999, con Gloria Bonder, quien desde sus investigaciones psicológicas sobre género y su activismo político desarrollado en la Argentina, ofrece una lectura minuciosa en su texto Género y subjetividad: avatares de una relación no evidente (Bonder, 1999). Este capítulo hace parte del libro Género y Epistemología: Mujeres y Disciplinas, en él se brinda un panorama de la problemática de género, a partir de los debates abiertos desde los años sesenta sobre liberación e identidad sexual; problematiza, además, en el concepto de género, dándole una amplitud que lo denomina como gran relato. La autora aborda conceptos como la genealogía de las concepciones de género, la crítica al binarismo género/sexo, para el análisis de la categoría de género, integra el abordaje histórico y filosófico más que contextual y situado.

El debate sobre género poco a poco se va encaminando a una consideración paulatinamente más social, tanto así que, en 2002, Ana Isabel Garay Uriarte publica la tesis doctoral Poder y subjetividad. Un discurso vivo, allí analiza la categoría de subjetividad desde una visión más foucaultiana y coincide con una idea del pensador francés, es en el cuidado de sí que es posible el acercamiento al sí mismo, tal como él lo sustento en la Hermenéutica del sujeto (Foucault, 1987). Garay interpreta al género como un aporte vivo, pues no simplemente se trata desde la subjetividad como una categoría de opresión, por medio del poder, sino que además, se considera una realidad construida y relacionada, a partir del contexto social. Su texto contribuye a encaminar las búsquedas de género y subjetividad en aras de una compresión situada.

Vale la pena mencionar, como se ha sostenido a lo largo de esta revisión, que el epicentro de la reflexión recientemente se encontraba en Estados Unidos, y en el tiempo en que se desarrollaba la investigación 
anteriormente mencionada se seguía pensando el género como un problema para la producción de subjetividad, así lo dice Hollway (2003) en su escrito Gender difference and the production of subjectivity, texto que integra la tercera parte del libro Discourse theory and practice, él desarrolla una reflexión a partir de la diferencia de género, una construcción de subjetividad acorde a la diferenciación ya nombrada, el género es concebido como un lugar en donde se pueden diferenciar posiciones y el coexistir.

Por ende, la reflexión en torno al género resulta una cuestión cada vez más compartida entre América Latina, Estados Unidos y Europa, porque sus problemas son universales y son abordados multidisciplinarmente, así lo resalta Sanucci (2005), en Una mirada sobre el género: la narración policial en la literatura: modos de construcción de la subjetividad de género en 'Caminaré en tu sangre', de Vicente Battista; desde un estudio literario analiza la subjetividad de género y en su relación intertextual incluye al discurso político, psicológico y crítico, en ese sentido, aporta un marco de referencia para pensar imaginarios sociales, representaciones, estereotipos, hibridaciones, configuraciones de sentido, pero fundamentalmente, demuestra cómo tanto la cultura popular como el género, obedecen a sistemas relacionales.

Es interesante notar también cómo en América Latina se van desarrollando cada vez más estudios sobre el género, poniéndolo en diálogo con ramas clínicas como el psicoanálisis, así lo revela el texto Psicoanálisis y género. La subjetividad de las diferencias entre los sexos, (Gutiérrez, 2005), la autora analiza a partir de la psicología la diferencia subjetiva que existe entre la construcción de sexos y géneros, desde el psicoanálisis lacaniano conversa con las teorías de género y la compleja creación de la subjetividad.

Los textos sobre subjetividad de género seguirán propiciando un debate más social, colectivo y contextual de la categoría, así se dan análisis como La subjetividad de género: el sujeto sexuado entre individualidad y colectividad (Cerri, 2010), en donde se propone analizar la validez analítica habida entre la dicotomía entre lo masculino y lo femenino como una construcción individual que termina afectando la acción social del sujeto y, por ende, la construcción de otras subjetividades. En esta misma línea está, Género, ciudadanía y derechos. La subjetividad politica y jurídica de las mujeres como clave para la igualdad efectiva (Esquembre, 2010). La autora pone como tarea de primer plano el principio de igualdad y no discriminación desde un punto de vista histórico el cual, aunque se ha pretendido en los textos constitucionales (en este caso España), se presenta como una constante la consagración de la desigualdad y se impide la igualdad a partir de la categoría objetiva de sexo masculino y femenino.

También encontramos estudios de género en Hispanoamérica, siendo un factor clave para la comprensión de la cultura patriarcal, la visión colonialista y decolonialista. Si para Paz (1989), América Latina es el resultado de una hostilidad que trae como consecuencia una particular soledad, para autoras como Segato (2014), las violencias patriarcales se sustentan en la ideología impuesta desde la colonia que se ha perpetuado a lo largo de varios siglos. Como consecuencia, resulta importante para la comprensión de género, investigar el pensamiento y las creencias en las que está sustentada la tradición, al igual que su incidencia en el lenguaje y en la lengua.

En comunidades de habla castellana, la concepción de género y subjetividad, implica revisar los aportes que vienen desde España, en donde en los últimos años abundan trabajos y publicaciones de género de gran interés como la Revista Duoda, fruto del Centro de Investigación de Mujeres de la Universidad de Barcelona. En el monográfico llamado Subjetividades de las mujeres (1992), se destaca el trabajo Aportaciones al debate del Diseño Curricular Base (Colectivo de la Escuela No sexista de Oviedo, 1992) en él, proponen que la educación no sexista sea un tema transversal a todas las áreas y contextos, porque ella puede propiciar el cambio de la mentalidad patriarcal, la jerarquía de roles y los estereotipos de género.

Sin duda, una educación de género puede ayudar a la prevención de sus problemáticas, contribuiría a disminuir la desigualdad en el proceso de construcción de la personalidad de niñas y niños, y en su evolución y desarrollo; de esta forma, los saberes que se transmiten en la escuela dejarían de ser androcéntricos. Es necesario desarrollar acciones positivas y tomar medidas correctoras de la desigualdad que viven las alumnas en los sistemas educativos, lo cual requiere una formación permanente del profesorado y de todos los 
profesionales de la educación, porque se trata de su futuro, para favorecer su formación profesional y la construcción equitativa de su propia subjetividad.

Así mismo, desde la península Ibérica viene desarrollando una importante labor el sello Editorial Horas y Horas de Madrid, nacido en 1991, quien se ha encargado de difundir el pensamiento femenino a nivel mundial. Una de sus tareas ha consistido en recuperar voces femeninas olvidadas, importantes para la reconstrucción de la subjetividad de género, entre los centenares de títulos publicados resalta Los cautiverios de las mujeres: madresposas, monjas, presas y locas (Lagarde, 1993), para la autora también la manifestación del amor femenino tiene que ver con el poder patriarcal. En otro texto, Género y feminismo. Desarrollo bumano y democracia (Lagarde, 1996), aclara lo que es 'La perspectiva de género' que, reconoce la diversidad de géneros y permite definir sus características:

La perspectiva de género tiene como uno de sus fines contribuir a la construcción subjetiva y social de una nueva configuración a partir de la resignificación de la historia, la sociedad, la cultura y la política desde las mujeres y con las mujeres. (Lagarde, 1996, p. 13)

Para Lagarde, el género es más que una categoría sociocultural, es una teoría que abarca categorías, fenómenos históricos, interpretaciones construidas en torno al sexo, relaciones, experiencias de vida, éticas, estéticas, políticas. En esta revisión se limita la búsqueda al análisis, a la teoría y la aplicación, así se encontró el artículo Subjetividad y esfera pública: el género y los sentidos de familia en las memorias de la represión (Jelin, 2011), la autora hace hincapié en la manera en que estas nociones culturalmente subjetivas se manifiestan en un pasado reciente. El análisis se sitúa en Argentina y toma como objeto de estudio una población vulnerable, similar a la de la investigación de la Universidad Santo Tomás (Jiménez, 2018), Jelin, debate sobre el género con base en casos concretos, brindando un modelo de interpretación desde la subjetividad de las memorias, los olvidos y los silencios, porque: "Se juegan allí afectos y sentimientos, que empujan a la reflexión y a la búsqueda de sentido” (Jelin, 2011, p. 556).

En cuanto a la construcción de género en la subjetividad infantil en el libro Sistema sexo/género, identidades y construcción de la subjetividad, (Martínez y Bonilla, 2010), en la primera parte se aborda la complejidad conceptual del concepto género que hace parte del conocimiento humano, de su diversidad y complejidad psicológica, al igual que de una percepción y constricción producto de una interacción cultural. Por tanto, la construcción es subjetiva, e implica abordar diversos procesos de sexuación y transformación. También exige el desarrollo de una teoría y de un modelo, capaz de medir cómo se ha construido la concepción de los tradicionales paradigmas de género. Se plantea, por ejemplo, analizar el modelo de androginia, las diferencias y conflictos de roles, las relaciones parentales y las de poder en un ámbito público y privado, las diferencias y semejanzas. Si bien la respuesta que ha dado la ciencia es amplia, es necesario seguir estudiando las problemáticas derivadas de la concepción de género, pues su visión es variable y compleja, además en su construcción, influyen variables que dependen del contexto.

Además, en la construcción de la individualidad no solo intervienen factores vinculados a variables de la persona y de la situación, sino que también influyen otros que -formando parte de ambas variables- presentan la peculiaridad de establecer categorías que inciden cognitivamente en las percepciones y atribuciones realizadas por los sujetos, tanto sobre sí mismos como sobre los otros, adscribiéndolos a grupos de pertenencia, lo que nos lleva a tomar en consideración como otro eje de análisis el binomio diferencias-semejanzas entre grupos. (Martínez y Bonilla, 2010, p. 10)

Todo esto complejiza más la aproximación de la categoría género que es también como ya se mencionó, una teoría, más si la limitamos a un contexto infantil, donde usualmente con base en el sistema de creencias y valores, se reproducen los modelos que existen en el hogar y en su entorno inmediato. En el estudio Implicancias de género en la construcción de la sexualidad adolescente, (Checa, 2013), la autora analiza la influencia de la familia, la cultura y la sociedad en la construcción de la subjetividad de género, pues existen imaginarios que se deben reinterpretar. Para este tipo de población de bajos recursos, el cuerpo forma parte esencial de su identidad, la adolescencia es compleja tiene cambios físicos, psicológicos y socioculturales, la 
definición de su identidad sexual es clave para su desarrollo y expectativas; de esta manera, la familia y el entorno social son definitivos para la subjetividad de género.

\section{Problemáticas de género en poblaciones infantiles}

\subsection{Género y subjetividad infantil}

Tal como se observó, definir género es problemático y se complejiza cuando se delimita a un grupo en particular. Los menores de edad están, por ejemplo, en plena construcción de su identidad de género, por tanto es altamente subjetiva su apreciación y lectura. A pesar de ello, se han detectado problemáticas propias de la niñez, en Configuración de la subjetividad en la primera infancia en un momento posmoderno (Espinosa, 2013), la pregunta central es ¿cómo se configura la subjetividad infantil en un grupo de niños y niñas de primera infancia de un jardín infantil en Bogotá? Acude al método narrativo e indaga en los modos se ser, sentir, decir, hacer y relacionarse de los niños consigo mismo y con su entorno; encuentra que el sujeto infantil es cambiante, influyen en él la sociedad y las experiencias que vivan, además, sus ideales muchas veces no coinciden con la realidad. Señala cómo el influjo de la cultura de masas y audiovisual es evidente, en esta medida, para comprender la subjetividad infantil es necesario conocer sus discursos, sus formas de comunicarse, de habitar y de relacionarse.

La subjetividad infantil está en constante transformación, se construye a través de la práctica y las condiciones socioeconómicas. "La subjetividad como un modo de hacer en el mundo, un modo de hacer con el mundo y un modo de hacerse en el mundo, es un modo de hacer con lo real y con la experiencia" (Espinosa, 2013, p. 19). Aquí se concibe al sujeto infantil como un ser en construcción, en donde es decisivo para su desarrollo su entorno cercano; el niño-la niña es producto de la intersubjetividad, es decir, del vínculo que establece con los otros. La autora demuestra también cómo la sociedad moderna domina en el discurso de los menores. El creciente consumismo convierte a la infancia en una entidad mediática. Este flagelo propio de la llamada posmodernidad, hace que los cuerpos infantiles ingresen en la realidad pública en donde predomina la imagen, esa es la nueva forma de subjetividad. Así, la subjetividad dominante está asociada a la razón instrumental, a la competencia y el éxito que excluye e ignora a muchos.

Del mismo modo, el problema del consumo invade también los espacios familiares, lo más grave es que existe la figura del adulto-niño, así más que las personas cuentan las imágenes que consumen; esto implica la aniquilación de la infancia. El capitalismo infantil transforma negativamente las relaciones humanas. Todo está inundado de discursos mediáticos, consumistas y patriarcales, mientras tanto los derechos de los niños y las niñas, que según la convención celebrada el 20 de noviembre de 1989 son universales (UNICEF, 2006), se van refundiendo para perpetuar y trasmitir lógicas e imaginarios culturales que reproducen la economía dominante, por ejemplo, universos simbólicos cargados de ideología, mundos que tienden a ser rosa o góticos, mundos que caracterizan la llamada felicidad juvenil. Espinosa (2013) concluye que es necesario indagar en la vida de los menores, en sus discursos y relaciones, interrogar sobre sus motivaciones, sobre cómo conciben los derechos, saber cómo habitan en su país, en su barrio, en su familia. Todo esto respondería el cómo configuran su subjetividad. Afirma que, en este momento de la historia, es preciso comprender cómo afecta en ellos la sociedad posmoderna y es necesario aprender a escucharlos y a conocer sus juegos, porque el juguete como producto social y cultural, seguramente será revelador. Igual sucede con ciertos acontecimientos de su vida como su cumpleaños, el Halloween, la Navidad. Estos hallazgos, también formaron parte de la discusión y elaboración del proyecto Yo soy tú, tú eres todos. Somos letras vivas en movimiento: un acercamiento lúdico infantil en perspectiva de género (Jiménez, 2018), allí los talleres implementados dieron cuenta de la subjetividad de género, donde surgieron problemáticas de percepción, reconocimiento, deseos, relatos violentos, palabras sexistas y diversas relaciones instrumentales de los niños del barrio Juan XXIII de Bogotá. 
Teniendo en cuenta la condición de países en vías de desarrollo, se encuentra que esta es una característica determinante a la hora de investigar sobre género. En el estudio 'Marginalization' in third world feminism: Its problematics and theoretical reconfiguration (Mansoor, 2016), el autor indaga en el desempoderamiento que concibe al hombre como centro y a la mujer como periferia, a esto se suma la condición de estar al margen de la economía mundial, característica propia de los países del tercer mundo. Este texto ayuda a comprender por qué en el factor económico influye en el desarrollo de género, se infiere que una niña de un contexto pobre será más vulnerable y tendrá menos oportunidades para desarrollarse.

The complexity of the sexes. Building sexual identity?(Lippe, 2016), sustenta que el desarrollo de la identidad sexual se relaciona con la identidad del individuo y posee varios dominios que interactúan entre sí: el biológico, el social y el psíquico, pues de ellos depende la evolución humana y el lugar que ocupa el género en la sociedad; aclara que la diversidad de género trae nuevos problemas. En este aspecto, el estudio de la Universidad Santo Tomás (Jiménez, 2018), contempló el componente sociocultural; desde la literatura se abordó la problemática de género a través de narrativas, lecturas, escrituras y diversas estrategias didácticas que permitieron identificar problemáticas de género en el mundo, en su país y en su contexto inmediato. Pero, esto no excluye que sería de gran interés, vincular también la lectura del fenómeno biológico y psíquico, lo cual requeriría de profesionales expertos en estas áreas. Un estudio que contempla el componente psicológico en la construcción de género es Ajuste psicológico según la edad y género en niños y jóvenes de estrato socioeconómico 1 y 2 de Bogotá (Henao y Mahecha, 2005) El objetivo es la prevención de la conducta agresiva en la población infantil y juvenil, para ello, estudia el comportamiento tanto a nivel externo e interno en niños y niñas de $4 \mathrm{a}$ 17 años, pertenecientes a un estrato socioeconómico bajo. Los resultados evidencian importantes diferencias de género, encontraron que los niños hombres tienen una conducta más externalizada y en ellos el problema de la agresividad es más prevalente que en las niñas.

Del trabajo anterior se deduce que, las pautas de crianza de los padres son decisivas para la construcción de la subjetividad de género, al igual que su situación económica, la edad y el manejo de las emociones y los afectos. En la población del proyecto: Yo soy tú, tú eres todos. Somos letras vivas en movimiento: un acercamiento lúdico infantil en perspectiva de género (Jiménez, 2018) participaron durante todo el proceso dos niños y catorce niñas, y aunque no se ejecutaron estudios psicológicos, si se confirman algunos hallazgos del estudio de Henao y Mahecha (2005) como la agresividad verbal en ambos géneros. La población estudiada, estratos 1 y 2 de Bogotá, reveló que la condición socioeconómica influye en la configuración de la subjetividad de los menores. En el estudio aún inédito de la Universidad Santo Tomás (Jiménez, 2018) se encuentra que, son los niños, quienes a pesar de ser minoría en el aula, presentaron mayor manifestación de agresividad física, lo cual lleva a pensar que este problema es más recurrente en este género; pero, también se detectó su existencia en algunas niñas, en un reporte de caso una respuesta a la pregunta: ¿A ti qué gustaría cambiar de ti misma?, fue, "Mi violencia. Soy muy agresiva con los hombres (...)" (Jiménez, 2018, p. 60). La problemática de la violencia infantil requiere profundización y prevención, porque si desde la niñez se reproduce, esto conduce a su normalización y trae como consecuencia más violencia y en ella, la mujer sigue siendo la principal víctima.

Otro problema relacionado con la construcción de género en la población infantil es el que se vive en el colegio. Construcciones de género y cultura escolar (Estrada, 2001), analiza las relaciones de género en este tipo de contextos. Desde un estudio etnográfico crítico, la autora trabaja en perspectiva de género, inicialmente asume prejuicios y preconcepciones de género existentes en el ambiente escolar e identifica roles, esto le implicó efectuar una vigilancia, especialmente a los dispositivos de poder que prescriben las identidades de género, lo cual también significó reconocer lugares de resistencia. Detectó juegos del lenguaje en los relatos de experiencias subjetivas, en donde el cuerpo formó parte del sí mismo (self), y cómo desde su estructura biológica influye en la cultura, así se reproducen modelos de masculinidad y feminidad, de sexualidad y familia, incluso de la psiquis. Este trabajo concibe el género como una especie de lente para mirar, analizar e interpretar lo que ocurre en el aula de clases, los actores escolares asumieron roles de género en distintas situaciones sociales, desde su contexto discursivo se interpretó su conducta. Este estudio aporta al análisis 
del contexto, identifica significados y recurrencias predominantes, esto lo logró gracias a la participación de talleres sobre su vida y sus relaciones con los demás, siendo la problemática de género el hilo conductor de la participación. Frente a los asuntos de género, la escuela influye notoriamente en el comportamiento y en la visión de los niños, la cultura escolar representa el imaginario de género predominante, pero también abre espacio para la diferencia. Pero, cómo tratarla en un mundo donde la globalización tiende a la homogeneidad.

Del mismo modo, la intersubjetividad de género influye en la visión de los menores, lo que ellos observan en su hogar y en su entorno es determinante; existen costumbres patriarcales arraigadas que se tienden a reproducir sin cuestionarlas, por ejemplo, asociar lo femenino a lo débil. De allí la importancia de integrar en el currículo la perspectiva de género desde la niñez, solo así es posible formar una visión crítica e incluyente, capaz de integrar la diferencia sexual y producir nuevos significados desde una cultura del respeto y valoración de lo femenino, género que tradicionalmente ha sido maltratado. Retomando el estudio Ajuste psicológico según la edad y género en niños y jóvenes de estrato socioeconómico 1 y 2 de Bogotá (Henao y Mahecha, 2005), se concuerda con la tesis de que en la escuela se construye una trama ecológica, que estructura lo local con lo social. Sus actores se convierten en agentes de socialización de género, allí se reproduce la cultura y es posible comprenderla y cambiarla. Además, existe un Currículum oculto (Torres, 1998), en el aula está contenida una violencia simbólica que se da por la desigualdad, y por unas relaciones de poder que imponen un modelo de género, una manera de ver y de sentir:

El trabajo pedagógico no solo contribuye a dar referencias sobre cómo debe ser interpretada la realidad, sino que también define itinerarios, formas y métodos de resolver los problemas que se plantean a los hombres y mujeres que poseen un mismo habitus. (Torres, 1998, p. 94)

El currículo oculto, en este caso hace referencia a la problemática de género que se vive en las aulas, por tanto, es importante investigarla y proponer pedagogías integradoras, que no desconozcan la experiencia real de los menores, sus discursos, sus miedos, sus sueños. Al respecto, los currículos oficiales tienen mucho camino por recorrer, pues los asuntos de género son transversales, se trata de formar un pensamiento y un lenguaje crítico, de propiciar relaciones de género desde una mirada flexible e integradora. Así, la subjetividad de género será desde la niñez un asunto importante para la vida, donde la identidad será el resultado de una construcción crítica visible en las conversaciones y relatos de los niños, de esta forma, los imaginarios femeninos y masculinos, serán un producto cultural que cuestione y no reproduzca acríticamente los modelos que la sociedad impone. Esta tarea no es fácil, más cuando desde hace siglos se lleva perpetuando un modelo patriarcal binario, pero, desde una pedagogía integradora y una didáctica lúdica, es posible comenzar a construir el cambio.

\subsection{Implementando nuevas pedagogías de género}

Para resolver los problemas de género en la niñez, existen nuevas miradas que afirman la necesidad de integrar una pedagogía del juego, así se sustenta Juego, niñez y género en la escolarización inicial. Reflexiones a partir de la capacitación docente (García y Bilinkis, 2013) Las autoras desarrollan su estudio en Buenos Aires, proponen capacitar a docentes sobre niñez, género y educación, para contribuir en el debate crítico sobre la desigualdad de género. Desde un tratamiento lúdico cercano a su experiencia antropológica, abordan la problemática de género. Encuentran que en el ambiente escolar existen concepciones de género que son necesarias de debatir, lo cual implica todo un proceso de reeducación, analizando cómo ha sido la intervención de los docentes frente a problemáticas concretas que presentan los menores de edad. En este sentido, el papel de la educación es fundamental, pero, es necesario que desde las mismas instituciones educativas se abran espacios de debate y de capacitación, para no seguir reproduciendo la historia de lo mismo, la historia de la opresión sexual que recae con todo su peso, más en el género femenino. 
Una respuesta a los viejos problemas de género como la violencia y la desigualdad es la que propone ¿Nuevas miradas a viejos problemas? El protagonismo del enfoque de derechos y el enfoque de género en las discusiones sobre pobreza y los programas sociales (Arcidiacono y Zibecchi, 2013) Los autores son conscientes de que se deben implementar políticas públicas las cuales den respuesta a la inequidad e injusticia de género, estas deben concebir al sujeto como un ser con derechos. Sin embargo, la realidad demuestra que estos se quebrantan y la pobreza lejos de menguar, crece y fomenta la desigualdad social. Y aunque países como Argentina presentan avances respecto a la política de género, es necesario conseguir más apoyo; además, proteger a las madres es fundamental para garantizar una infancia feliz. Desafortunadamente las tareas domésticas no se visibilizan lo suficiente y siguen recayendo en las mujeres, esta realidad la perciben los niños y las niñas, porque desde su casa existe la desigualdad en el reparto de tareas.

De otro lado, también es recomendable impulsar acciones de autocuidado y cuidado de género, esto resulta esencial para el cumplimiento de derechos, así se garantiza un bienestar familiar y social. Y aunque en la actualidad casi todos los países hayan incrementado políticas de género, mientras exista la pobreza material y escolar, no será posible lograr un cambio de mentalidad.

El enfoque de derechos es crucial, pero no suficiente si no se acompaña de acciones que resuenen desde la academia. Arcidiacono y Zibecchi (2013) sostienen que, si no se logra una alfabetización de género, difícilmente se cumplirán los derechos, además las mujeres en condiciones de pobreza están más desprotegidas y por tanto, serán presa fácil de diversos tipos de violencia, lo cual sin duda afectará a su familia y especialmente a los menores, por lo que propiciar su autonomía económica será de suma importancia. Todo esto implica revisar legislaciones, currículos, teorías existentes, para restablecer al ser humano como un sujeto de derechos, sin desigualdad ni discriminación negativa.

Los asuntos de género no son solo una responsabilidad individual sino social y política. La pobreza femenina y las poblaciones vulnerables como los niños y las mujeres son multicausales. Existen causas de carácter afectivo como las separaciones y divorcios, en su mayoría sustentados en problemas de género, embarazos adolescentes, hogares monomaternales de mujeres cabeza de familia que para subsistir ejecutan trabajos en condiciones desfavorables, acceden a empleos informales, tienen bajos salarios, ganan menos que los hombres. Esto trae como consecuencia mayor empobrecimiento femenino, realidad que se transmite en el hogar. ¿Cómo viven las mujeres? ¿Cómo es la educación de los menores? Responder estos interrogantes será importante para conocer la vida familiar de las personas menores. Para Arcidiacono y Zibecchi (2013), una educación con más enfoque de derechos y enfoque de género, garantizaría el disminuir gran parte de su problemática, esta sería una forma de combatir la pobreza, pues la igualdad de género no es un tema menor sino urgente de atender. El gran problema sigue siendo el cambio de mentalidad patriarcal que, está presente en todas las esferas de la cultura y sigue reproduciéndose en la niñez y la juventud. Existen muchos estudios que, como se ha visto, impulsan el cambio, pero el compromiso debe ser mayor, debe involucrar a todas las instancias económicas, políticas y culturales del mundo. Se deben garantizar políticas a favor de la mujer.

Pero, desafortunadamente en los países latinoamericanos es evidente la desigualdad educativa, así lo demuestra el estudio comparado Desigualdad educativa, niñez y género. Educación comparada entre España y México (Lera, Ochoa, Cantú, Rivas y Martínez, 2017), revela que la desigualdad incrementa todo tipo de problemas. Los autores comparan la educación de dos países que tienen en común la misma lengua, credo, ideología patriarcal y políticas educativas semejantes; analizan el mestizaje como producto de la colonización. También revelan la existencia de varias problemáticas las cuales afectan, como la deserción escolar y la desigualdad, estas tienen como raíz la pobreza económica, por eso superarla constituye el gran reto. A diferencia de lo que ocurre en España, en el contexto mexicano existe una educación más inclusiva, intercultural y bilingüe, pero a pesar de los esfuerzos de inclusión educativa, aún existe la desigualdad de género. Los resultados demostraron que, es necesario para los dos países seguir invirtiendo en políticas públicas y presupuestos para lograr la equidad de género. Recuerdan que las estrategias de apoyo a la niñez y el género, de acuerdo al Banco Mundial, se basan en cuatro objetivos, indispensables para lograr un mundo más 
igualitario: a) Reducir la mortalidad materna y cerrar las brechas que subsisten en salud y educación. b) Crear más y mejores empleos para las mujeres y los hombres. c) Disminuir la desigualdad de género relacionada con la propiedad y el control de los activos clave como la tierra, la vivienda, la tecnología y el financiamiento. d) Mejorar la capacidad de las mujeres para hacerse oír y dirigir sus propias vidas. (Banco Mundial, citado por Lera et al., 2017). Lograr la equidad de género es un desafío de toda la sociedad, y es desde la educación y la población infantil donde debe promoverse el cambio.

\subsection{Narrativas e historias de vida}

$\mathrm{Al}$ revisar textos en torno a acontecimientos de género con base en narrativas e historias de vida, $E l$ enfoque biográfico: su validez metodológica, sus potencialidades (Bertaux, 1999), muestra desde el punto de vista sociológico la coyuntura actual entre en el pluralismo de las teorías y los métodos presentes en las investigaciones cualitativas pues, desde el punto de articulación entre ambas se pretende encontrar los puntos de inflexión entre las dinámicas históricas y las diversas manifestaciones sociales. En 2002 dos textos asumen la biografía como un método válido para emprender el análisis de la vida. El primero, El estudio de caso como informe biográfico-narrativo escrito por Botía (2002), hace una revisión de fuentes desde una forma particular de relato. El segundo, Los estudios de caso en la lógica de la investigación interpretativa de Pérez, Pozo y Fernández (2002), resulta más revelador a la hora del entendimiento de los hechos sociales, culturales, jurídicos, arqueológicos, etnográficos psicológicos o educativos. De tal manera que, la narración es aceptada como una forma de análisis, a partir de la biografía y la interpretación.

Por su parte, el artículo El método de estudio de caso: estrategia metodológica de la investigación cientifica (Martínez, 2006), orienta el desarrollo del método de estudio de caso a partir del correcto uso de los conceptos de valor, beneficio y utilidad práctica, a la luz de los debates que existen de si este método puede o no ser analizado a partir de las categorías epistemológicas de fiabilidad, validez y veracidad. Demuestra que el estudio de caso es una manera de abordaje investigativo más validada, pero usada únicamente en investigación cualitativa y en pocos momentos -como lo veíamos en el artículo de 1999-, asume la narración o la construcción literaria. El informe de investigación con estudios de casos (Ceballos, 2009) amplía la manera de ver la construcción de relatos, analiza la importancia de dar a conocer sus hallazgos, así como la dificultad para definir las características de estos informes. En otro texto La investigación con relatos de vida: Pistas y opciones de diseño metodológico, (Cornejo, Mendoza y Rojas, 2008), se demuestra que existe una articulación entre las experiencias particulares con sus respectivos significados y las prácticas sociales, bajo la luz de lo biográfico, sugiere modos de abordar los relatos de vida, donde afloran casi siempre representaciones tradicionales de género.

En Los estudios de casos y el problema de la selección de la muestra. Aportes del sistema de matrices de datos (Kazez, 2009), se otorga importancia a la articulación y coherencia de los momentos investigativos. El autor se centra en cuestiones de muestreo y resalta que el Sistema de Matrices de Datos es una herramienta de utilidad la cual justifica metodológicamente la decisión de la muestra. También Técnicas conversacionalespara la recogida de datos en investigación cualitativa: La historia de vida, (Meneses y Cano, 2008) indica cómo se deben construir las historias y los relatos de vida, cómo hacer el análisis y la interpretación del material biográfico y cuáles son las potencialidades y limitaciones de esta técnica conversacional. Sobre las historias de vida en Investigación Cualitativa (Balcázar, González, Gurrola y Moysén, 2013) se considera que cada etapa de la vida tiene un significado y una asociación con el tipo de familia en donde se establecen roles, jerarquías y alianzas. Otro texto, Historias de vida: una metodología de investigación cualitativa (Chárriez, 2012), presenta y discute las principales fases y estrategias utilizadas en las historias de vida, explica las rutas estructurales, así como las maneras como se han utilizado. Esta misma idea está en Una propuesta para el estudio de la identidad con aportes del análisis narrativo (Capella, 2013), aquí se estudia la identidad personal narrativa desde una perspectiva constructivista, con base en autobiografías escritas y entrevistas en el formato de relatos de vida en 
el campo de la psicología clínica. En este sentido, las historias de vida pueden revelar la subjetividad, a través de relatos sobre género que permiten identificar representaciones predominantes. En la investigación de la Universidad Santo Tomás (Jiménez, 2018) fue posible que los niños contaran de diferentes formas, a través de relatos, dibujos, juegos, archivos personales como objetos, fotos, juguetes, algunos momentos relevantes de su vida que permitieron identificar roles de género, relaciones de poder, afinidades afectivas y también sueños.

Mujeres y hombres ¿qué tan diferentes somos? Manual de sensibilización en perspectiva de género (Instituto Jalisciense de las Mujeres, 2008), es una herramienta conceptual y metodológica para comprender la perspectiva de género, para identificar algunas de sus problemáticas y promocionar la equidad sexual. Similar a la investigación de la Universidad Santo Tomás (Jiménez, 2018), la estrategia lúdica es el taller que busca trascender la predominante concepción patriarcal y visibilizar a cada hombre y a cada mujer desde diferentes dimensiones, biológica, histórica, social, cultural. Además, explora una dimensión explicativa la cual contribuya a la construcción de género equitativa e incluyente. Identifica características y diferencias sexuales, culturales, estereotipos que influyen en la identidad y la igualdad, a la vez que analiza lo contemplado en las legislaciones existentes. Este manual ayuda a pensar las relaciones de género para mejorar las relaciones humanas y producir un cambio de mentalidad que permita avanzar hacia una sociedad más igualitaria.

En la publicación Hacer los derechos realidad. Taller de sensibilización sobre cuestiones de género (Amnistía Internacional, 2007), se presentan dos módulos con el fin de concienciar sobre los problemas de género, enfatizando en los Derechos Humanos. El primero trata sobre temores relacionados con el género, el segundo sobre algunos mitos y Derechos. Este texto incluye todos los talleres a fin de que se puedan replicar en diversos ambientes y geografías. Así mismo, en América Latina se dio a conocer la Guía didáctica para trabajar género y masculinidad con niños, niñas y adolescentes titulada Creciendo Juntos y Juntas publicada por la Red de Masculinidad por la Igualdad de Género (REDMAS) (2007), que trata sobre la igualdad de género desde la Infancia, y fue creada en el marco del Programa Creciendo Juntos de Save The Children. La guía reúne a varias organizaciones de Nicaragua interesadas en trabajar asuntos de género, principalmente relacionadas con la construcción de masculinidades, tomando como referencia varias edades y condiciones sociales. Su objetivo es prevenir la violencia masculina, la guía afirma la importancia de hablar sobre género desde temprana edad, porque

Los niños y las niñas quedan sujetos desde muy temprana edad a las normas que definen lo 'masculino' y lo 'femenino'. A los varones se les dice que no deben llorar, que no deben sentir temor, que no deben ser sensibles, y que deben ser enérgicos y fuertes. A las niñas, por otro lado, se les dice que no sean exigentes, que sean sensibles, que sean complacientes y que se comporten como damas. (REDMAS, 2007, p. 9)

REDMAS (2007) confirma que muchos comportamientos de género negativos se originan en la niñez, por consiguiente, para evitar que sean víctimas de la violencia basada en género como la discriminación, es necesario promover el empoderamiento de esta población, el conocimiento de sus derechos, esto se logra con la valoración de sus relatos de vida, así es posible producir un efecto catártico y transformador. La guía ofrece ejercicios prácticos para pensar sobre el género, aborda también ejercicios sobre sexualidad, violencia de género y discriminación, al igual que la explotación sexual comercial. Como se puede constatar, para implementar una estrategia en perspectiva de género es fundamental el conocimiento de las historias de vida, porque "La historia de vida pretende atribuir a la subjetividad un valor de conocimiento dado el surgimiento y preponderancia de los métodos cuantitativos” (Balcázar et al., 2013, p. 13).

\section{Conclusiones}

Es importante que la educación trabaje desde la niñez la perspectiva de género, para contribuir a la construcción de un sujeto libre, autónomo, respetuoso de los Derechos Humanos y la diferencias, porque más que una categoría biológica, el género se determina por la cultura. En la niñez está estrechamente vinculado 
con la familia y los entornos inmediatos, por lo que es substancial conocer su contexto a partir de sus propias narrativas, relatos y acontecimientos e historias de vida.

Es prioritario seguir investigando en torno a la subjetividad de género desde la niñez, no solo para detectar en forma temprana sus problemáticas y evitar así la reproducción del feminicidio, sino también para educar desde el autocuidado de sí, la construcción de uno mismo como fin, tal como lo entendió Foucault (1987) Esto se logra implementando pedagogías y didácticas contextualizadas, lúdicas y en perspectiva de género. Uno de los expertos en didáctica de la lengua y la literatura Bombini (2015), afirma que es preciso enseñar y aprender la lengua y la escritura en contextos de vulnerabilidad social, porque es importante "entender a los sujetos que participan en ella [para propiciar] relaciones entre ellos y el mundo del conocimiento" (p. 41). En este sentido, cada entorno es singular, y aunque existan condiciones comunes que caracterizan a aquellos considerados como pobres, una constante es que en ambientes desfavorecidos económicamente se recrudece aún más la violencia contra las mujeres.

Pero, cada contexto puede presentar además de problemas, espacios de creatividad, de memoria, de crítica al sistema. En la investigación desarrollada por la Universidad Santo Tomás (Jiménez, 2018) se encontró que la mayoría de los niños participantes pertenecen a estratos 1 y 2 y viven en inquilinato, su percepción de esta realidad es positiva porque la relación con los otros se reconoce desde la cooperación y la solidaridad. Todos son vecinos y en un momento dado se convierten en una especie de familia que, sirve de protección frente a la realidad externa que la mayoría percibe con miedo porque saben que existe la violencia y diversas formas de agresión, donde identifican que las niñas son más vulnerables.

En la misma línea, es pertinente comprender la transformación que ha sufrido la sociedad, la familia, el mundo, no solo por el fenómeno de la tecnología, sino además, en lo concerniente a los asuntos de género, lo cual ha traído como consecuencia una paulatina apertura a la diversidad sexual y a la superación de los estereotipos tradicionales. Por otro lado, está la transformación que afecta a la misma naturaleza, propiciada por la creciente razón instrumental que parece reducir todo a objeto de consumo, realidad que afecta principalmente a la mujer, cuya existencia se intenta mercantilizar.

De igual modo, se convierte en un imperativo volver al humanismo, valorizar por ejemplo, el papel de la madre como educadora desde el afecto es una tarea que no debe desdeñarse, más cuando parece que la sociedad actual se olvida de vincularla como sabedora y generadora de pedagogía amorosa. Pero, ¿qué lugar le da la sociedad? Se podría decir que una de las tareas de género prioritaria es incluir y reconocer la enorme tarea e influencia de las mujeres en la construcción de cada individuo, ellas constituyen e influyen su universo e historia inmediata, su rol es decisivo para la formación de cada ser humano y de la sociedad. Igualmente, es recomendable abordar la reflexión en torno a las nuevas formas de feminidad, masculinidad y diversidad sexual, frente a ellas ¿cómo educar a los niños?

Consideramos además que, si se desea cambiar la historia de las representaciones patriarcales, la historia del poder según Foucault (1994) y Segato (2010), es necesario que desde la niñez se implemente la lectura crítica del entorno. Porque si se logra sensibilizar y reconocer sus problemáticas, será posible comenzar a transformar la realidad y los imaginarios de poder que afectan a las niñas y a las mujeres, y de esta manera, se promoverá un trato no violento que puede contribuir a la erradicación del feminicidio. También se lograrán establecer relaciones más dialógicas, incluyentes y horizontales.

Según la investigación referida que dio pie a esta revisión, la de la Universidad Santo Tomás (Jiménez, 2018), la subjetividad en los menores tiende a ser homogénea, sus relatos reflejan el predominio de la cultura patriarcal, sus relaciones inocentemente reproducen la ideología dominante que es visible en su expresión verbal y gestual. Transformar los imaginarios de poder que asocian lo femenino a lo débil y lo masculino a lo fuerte no es fácil, sin embargo, se detectó que cuando los niños tienen la posibilidad de contar y socializar sus historias, tienen la posibilidad de cuestionarlas y de aprender a través de la propia experiencia y la de los otros. De esta manera, trabajar desde su propia vida y entorno se convirtió en esencial para generar reconocimiento, pertenencia, afecto, lo que se tradujo en relaciones con el otro más respetuosas y flexibles. Sin el otro, niño, 
madre, padre, maestro... no es posible generar cambios cualitativos. De aquí se deduce que la identidad de género no solo tiene que ver con la equidad o los derechos, sino con toda la cultura, pues existen visiones erróneas las cuales afectan negativamente a las mujeres. Segato (2010) sostendrá que precisamente lo que hay que transformar es la ficción dominante que asocia lo femenino a lo débil o a la víctima, ficción que subsiste aún en el siglo XXI.

Como aquí se observó, en la sociedad actual existen diversas formas de violencia contra las mujeres que tienden a normalizarse, una de las más terribles es el feminicidio, otra el uso y el abuso de su cuerpo, por eso la educación de género debería ser algo continuo y orientada especialmente a la población infantil. Esto implica una revisión del mundo y sus imaginarios en torno a lo que entiende por ser hombre o ser mujer. Desafortunadamente existe como lo piensa Butler (2007) una racionalidad de género instaurada que es preciso deconstruir, esto se puede lograr fomentando el reconocimiento de la propia vida.

Esta revisión confirmó que la educación, la pedagogía unida a estrategias didácticas lúdicas y los afectos, son factores de protección y prevención de las violencias basadas en género. Confirma además que cada sujeto es un ser único, dueño de una subjetividad que comienza a construirse desde la niñez, desde esta etapa se configurará lo que será su existencia en el mundo y sus relaciones con los demás, de allí que requiera todo el apoyo y cuidado de la sociedad. ¿Es esto posible?

Es conveniente erradicar los índices de pobreza económica y social porque de allí nace la población vulnerable y el incremento de los problemas de género. Pero las problemáticas de género son multicausales y trascienden las condiciones económicas y sociales. En este sentido, la educación como se ha resaltado, representa un gran factor de protección humana y cambio cultural positivo y, si se desea construir sociedades más equitativas e incluyentes, sin duda ella debe integrar la perspectiva de género e impactar desde la niñez, de esta manera se garantizará un futuro con menos feminicidios.

\section{ReFERENCiAS}

Amnistía Internacional (2007) Hacer los derechos realidad. Taller de sensibilización sobre cuestiones de género. Recuperado de http://bit.ly/2ySAaWw

Arcidiacono A. del P. y Zibecchi, C. (2013) ¿Nuevas miradas a viejos problemas? El protagonismo del enfoque de derechos y el enfoque de género en las discusiones sobre pobreza y los programas sociales. Voces en el Fenix, 4(23), 118-125. Recuperado de http://bit.ly/2KSIWL9

Balcázar P., González, N., Gurrola G. y Moysén A. (2013) Investigación Cualitativa. México: Universidad Autónoma del Estado de México.

Beauvoir, S. (1999) El segundo sexo. Los hechos y los mitos. Madrid: Cátedra, D.L.

Beauvoir, S. (2000) El segundo sexo. Vol. 2. La experiencia de vida. Madrid: Cátedra.

Bertaux, D. (1999) El enfoque biográfico: Su validez metodológica, sus potencialidades. Proposiciones, (29), 1-23.

Bombini, G. (2015) Textos retocados. Lengua, literatura y enseñanza. Buenos Aires: Ediciones el Hacedor.

Bonder, G. (1999) Género y subjetividad: Avatares de una relación no evidente. En: S. Montecino y A. Obach (Eds), Género y Epistemologia: Mujeres y Disciplinas (pp. 29-55) Santiago de Chile: Universidad de Chile.

Botía, A. B. (2002) El estudio de caso como informe biográfico-narrativo. Arbor, 171(675), 559-578.

Butler, J. (2007) El género en disputa. El feminismo y la subversión de la identidad. Barcelona: Paidós.

Butterfield J. (2015) Fowler's Dictionary of Modern English Usage, Oxford, 4taed. Recuperado de http://bit.ly/2Ttk $\mathrm{P} 8 \mathrm{w}$

Capella, C. (2013) Una propuesta para el estudio de la identidad con aportes del análisis narrativo. Psicoperspectivas, 12(2), 117-128.

Caracol. (8 diciembre, 2016) El crimen de Juliana Samboni minuto a minuto. Bogotá, Colombia. Recuperado de htt p://bit.ly/2KDyt $4 \mathrm{~J}$ 
Cardona G. P. y Onofre D. (2017) El feminicidio y las representaciones sociales de ser mujer. Forensis 2017. Datos para la vida. Bogotá: Instituto Nacional de Medicina Legal y Ciencias Forenses.

Ceballos, F. (2009) El informe de investigación con estudio de casos. Magis. Revista Internacional de Investigación en Educación, 1(2), 413-423. Recuperado de http://bit.ly/2KCJZxo

Cerri C. (2010) La subjetividad de género: el sujeto sexuado entre individualidad y colectividad. Gaceta de Antropología, 26(2). Recuperado de http://bit.ly/2MbxoEF

Colectivo de la Escuela- No sexista de Oviedo (1992) Aportaciones al debate del Diseño Curricular Base, Revista Duoda: estudis de la diferència sexual, (3), 189-194.

Cornejo. M., Mendoza, F. y Rojas, R. (2008) La investigación con relatos de vida: Pistas y opciones de diseño metodológico. Psykhe, 17(1). Recuperado de http://bit.ly/300NQuN

Chárriez, M. C. (2012) Historias de vida: Una metodología de investigación cualitativa. Revista Griot, 5(1), 50-67.

Checa S. (2013) Implicancias de género en la construcción de la sexualidad adolescente. Buenos Aires: Dirección General de Cultura y Educación de la Provincia de Buenos Aires.

Espectador. (20 de agosto, 2017) Capturan a excomisaria de familia de Armero, por caso de Sara Salazar. Bogotá, Colombia. Recuperado de http://bit.ly/2Z1lvTP

Espectador. (26 de noviembre, 2018) Gobernadora del Meta pide cadena perpetua para asesino y violador de una niña de seis años. Recuperado de http://bit.ly/31t93xF

Espinosa, A. M. (2013) Configuración de la subjetividad en la primera infancia en un momento posmoderno. Infancias Imágenes, 12(2), 18-28. Recuperado de http://bit.ly/2z35gep

Esquembre M del M. (2010) Género, ciudadanía y derechos. La subjetividad política y jurídica de las mujeres como clave para la igualdad efectiva. Anuario de Derecho Parlamentario, (23), 47-85. Recuperado de http://bit.ly/2T kW52a

Estrada, A. M. (2001) Construcciones de género y cultura escolar, Los fragmentos del calidoscopio. Una propuesta teórico-metodológica para el análisis cualitativo de las relaciones de género en la escuela. Revista Nómadas (14). Recuperado de https://www.redalyc.org/pdf/1051/105115268002.pdf

Foucault, M. (1987) Hermenéutica del sujeto. Madrid: Ediciones La Piqueta.

Foucault, M. (1994) Microfisica del poder. Barcelona: Planeta-Agostini.

Garay, A. I. (2002) Poder y subjetividad un discurso vivo. Barcelona: Universitat Autónoma de Barcelona.

García M. I. y Bilinkis M. (2013) Juego, niñez y género en la escolarización inicial. Reflexiones a partir de la capacitación docente, Revista Lúdicamente, 2(4), Buenos Aires. Recuperado de http://bit.ly/2ZQeVQZ

Gutiérrez M. (2005) Psicoanálisis y género. La subjetividad de las diferencias entre los sexos, Convergencia, 12(37), 139-168.Recuperado de http://bit.ly/2ToQ6cC

Henao M. y Mahecha J. C. (2005) Ajuste psicológico según la edad y género en niños y jóvenes de estrato socioeconómico 1 y 2 de Bogotá, Publicaciones Konrad Lorenz, 12(2), 197-212. Recuperado de http://bit.ly/2 TpHQJp

Heredia, D. C. (2015) Violencia intrafamiliar contra niñas y adolescentes mujeres en Colombia, 2009-2014. Bogotá: Instituto Nacional de Medicina Legal y Ciencias Forenses.

Hollway, W. (2003) Gender difference and the production of subjectivity. En M. Wetherell y S. Taylor, Discourse Theory and Practice (pp. 272-283). London, United Kingdom: Sage Publications.

Instituto Jalisciense de las Mujeres (2008) Mujeres y hombres ¿Qué tan diferentes somos? Manual de sensibilización en perspectiva de género. México: Instituto Jalisciense de las Mujeres. Recuperado de http://bit.ly/2Z0H3DK

Instituto Nacional de Medicina Legal y Ciencias Forenses [INMLCF]. (2017). Forensis. Datos para la vida. Bogotá: Imprenta Nacional de Colombia.

Jelin, E. (2011) Subjetividad y esfera pública: El género y los sentidos de familia en las memorias de la represión. Politica y Sociedad, 48(3), 555-569.

Jiménez, M. (2018) Yo soy tú, tú eres todos. Somos letras vivas en movimiento: un acercamiento infantil en perspectiva de género. Bogotá: Universidad Santo Tomas, Facultad de Filosofía y Letras, Convocatoria Fodein. 
Kazez, R. (2009) Los estudios de casos y el problema de la selección de la muestra: Aportes del sistema de matrices de datos. Subjetividad y Procesos Cognitivos, 13(1), 71- 89.

Lagarde, M. (1996) El género. En Género y feminismo. Desarrollo humano y democracia. Madrid: Ed. horas y HORAS.

Lagarde, M. (1993) Los cautiverios de las mujeres: madresposas, monjas, presas y locas. México: Universidad Autónoma de México.

Lera J. A., Ochoa R. F., Cantú D., Rivas J. I. y Martínez J. C. (2017) Desigualdad educativa, niñez y género. Educación comparada entre España y México. Málaga: Universidad de Málaga.

Lippe D. (2016) The complexity of the sexes. Building sexual identity? Enfances \& Psy, 69(1), 27-37. Recuperado de http://bit.ly/2MaPHd3

Lozano, N. (2018) Comportamiento del homicidio, Colombia 2018. Forensis 2018.Datos para la vida. Bogotá: Instituto Nacional de Medicina Legal y Ciencias Forenses. Recuperado de http://bit.ly/2TlSH7b

Mansoor, A. (2016) "Marginalization" in third world feminism: Its problematics andtheoretical reconfiguration. Palgrave Comunications, 2(16026). Doi: https://doi.org/10.1057/palcomms.2016.26

Martínez I. y Bonilla A. (2010) Sistema sexo/género, identidades y construcción de la subjetividad. Valencia: Universitat de Valencia.

Martínez, P. C. (2006) El método de estudio de caso estrategia metodológica de la investigación científica. Rev. Cientifica Pensamiento y Gestión, (20), 165-193, Barranquilla: Universidad del Norte.

Meneses M. T. y Cano A. (2008) Técnicas conversacionales para la recogida de datos en investigación cualitativa: La historia de vida. Nure Investigación, (37), 1-6.

ONU MUJERES (2018) Recomendaciones generales adoptadas por el Comité para la Eliminación de la Discriminación contra la mujer. Recuperado de http://bit.ly/2Tkz4w4

Paz, O. (1989) El laberinto de la soledad. España: Fondo de Cultura Económica.

Pérez, J., Pozo, T. y Fernández A. (2002) Los estudios de caso en la lógica de la investigación interpretativa. Arbor, 171(675) 533-557.Recuperado de http://bit.ly/2ZPQVNW

Presidencia de la República (2015) Ley 1761 de 2015. Recuperado de http://bit.ly/2YU2QJj

Radford J. y Rusell D. (1992) Femicide. The politics of Woman Killing. New York: Twayne Publishing/Macmillan Publishing Company. Recuperado de http://bit.ly/2YHB080

Real Academia Española [RAE] (2019) Diccionario de la Lengua Española, versión online. Recuperado de http://ww w.rae.es/

Red Colombiana de Mujeres Científicas [RCMC]. (2018) Red Colombiana de Mujeres Cientificas. Recuperado de h ttp://bit.ly/2GZM3OW

Red de Masculinidad por la Igualdad de Género [REDMAS]. (2007) Guía didáctica para trabajar género y masculinidad con niños, niñas y adolescentes: Educando en igualdad materiales para trabajar en el Aula. Recuperado de http://bit.ly/2H0IE2x

Rodríguez D. J. y Jiménez l. (2018) Comportamiento de la violencia intrafamiliar, Colombia año 2018. Forensis 2018. Datos para la vida. Bogotá: Instituto Nacional de Medicina Legal y Ciencias Forenses. Recuperado de http:// bit.ly/2TlSH7b

Sanucci, M. E. (2005) Una mirada sobre el género: La narración policial en la Literatura. Question, 1(8), 1-9. Recuperado de http://bit.ly/2Mdp2MQ

Scott, J. (1990) El género una categoría útil para el análisis histórico. En J. Amelang y M. Nash (Ed), Historia y género: Las mujeres en la Europa Moderna y Contemporánea (pp. 265-302). Valencia: Edición Alfons El Magnànim, Institució Valencina d Estudis i Investigació.

Segato, R. L. (2010) Las estructuras elementales de la violencia. Buenos Aires: Universidad Nacional de Quilmes.

Segato, R. L. (2014) Colonialidad y patriarcado moderno: expansión del frente estatal, modernización, y la vida de las mujeres. En: Y. Espinosa, D. Gómez y K. Ochoa (Ed), Tejiendo de otro modo: Feminismo, epistemología y apuestas decoloniales en Abya Yala(pp.75-90). Popayán: Editorial Universidad del Cauca.

Torres, J. (1998) El curriculum oculto. Madrid: Ediciones Morata S. L. 
Myriam Jiménez Quenguan, et al. La necesidad de educar en perspectiva de género

UNICEF (2006) Convención sobre los derechos del niño. 20 de noviembre de1989. Madrid: Unicef Comité Español. Recuperado de http://bit.ly/2MiXoOz

Witt, S. D. (1997) Parental influence on children's socialization to gender roles. Adolescence, 32(126), 253-260.

Zemon, N. (1975), Women's History in Transition: The European Case. Feminisi Studies, (3), 83-103

CC BY-NC-ND 